Article

\title{
Comparative Study of Stator Configurations of a Permanent Magnet Linear Oscillating Actuator for Orbital Friction Vibration Actuator
}

\author{
Jianhui Hu *, Meng Zhao, Jibin Zou and Yong Li \\ School of Electrical Engineering and Automation, Harbin Institute of Technology, Harbin 150006, China; \\ zhaomeng@hit.edu.cn (M.Z.); zoujibin@hit.edu.cn (J.Z.); liyong611@hit.edu.cn (Y.L.) \\ * Correspondence: hujianhui@hit.edu.cn
}

Academic Editor: Stanislaw M. Dubiel

Received: 8 April 2017; Accepted: 5 June 2017; Published: 17 June 2017

\begin{abstract}
A PM orbital friction vibration actuator (OFVA) which composes four linear oscillating actuators (LOA) is proposed in this paper. This paper presents the design, analysis, and experimental validation of stator configuration of a permanent magnet LOA to improve its thrust force characteristics. First, the magnetized topology and the coil configuration are interpreted. The optimization design goal of the LOA was established and the end effects of the actuator are illustrated. The influences of stator design parameters on the performance of LOA were investigated and the optimal parameters have been identified with reference to the thrust force density and thrust force ripple. Results showed that a quasi-Halbach magnetized E-cored LOA with obtrapezoid teeth has the best electromagnetic performances of all the LOAs examined here. Finally, the predicted thrust force characteristics were validated by measurements on a prototype actuator.
\end{abstract}

Keywords: linear oscillating actuator; permanent magnet actuator; stator configuration; thrust force; orbital friction vibration actuator

\section{Introduction}

Orbital friction vibration actuators (OFVA) have become a core part of orbital friction welding technology whose applications fall into many industries [1-4]. As the force-supply unit of the orbital friction welding machine, the OFVA should generate higher thrust force when it operates in relatively high frequency. A permanent magnet (PM) OFVA composed of four identical PM LOAs and springs is proposed, as shown in Figure 1, for orbital friction welding machine. The performance of the OFVA is mostly determined by the thrust force characteristics of the LOAs. The parameters of LOA have a significant influence on the thrust force and its fluctuation. In this paper, the stator configurations of PM LOAs are thoroughly investigated to increase the force and suppress the fluctuation.

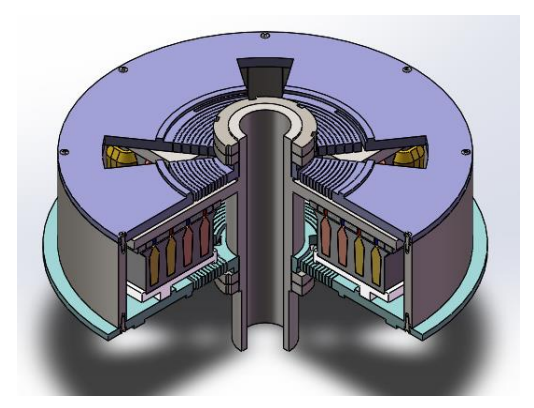

Figure 1. Proposed a permanent magnet (PM) orbital friction vibration actuators (OFVA). 
There are two LOAs distributed in the $\mathrm{x}$ direction and two more in the $\mathrm{y}$ direction of the OFVA to control motion in $\mathrm{x}$ and $\mathrm{y}$ directions, separately. Due to the decoupled relationship between $\mathrm{x}$ and $\mathrm{y}$ direction movement, only one LOA was here analyzed.

The thrust force ripple is an important parameter of LOA, key to ensuring the smooth operation. The thrust force ripple is affected by the end effect common existed in linear motors, and it is also affected by the magnetic circuit structure of LOA. The thrust force ripple needs to be modeled and reduced.

The thrust force density is another important parameter of LOA. At present, the thrust force per area of LOA can be $48,360 \mathrm{~N} / \mathrm{m}^{2}$ [5], the linear motor can reach $83,000 \mathrm{~N} / \mathrm{m}^{2}[6,7]$ by optimization. A design method was proposed for unit LOA to maximize thrust force density in [8], the quasi-Halbach magnetized unit LOA can reach $112,700 \mathrm{~N} / \mathrm{mm}^{2}$, while it focus on the comparative design of the PM mover, and the stator is still needed to design and analysis.

To improve the performance of OFVA, the design principles and the structural characteristics of the E-cored PM LOA are illustrated in this paper. The influences of the end effects associated with the finite length armature and stator's design parameters are also investigated. The main design parameters of the actuator and the shape of the teeth and slot were designed to maximize thrust force density while minimizing thrust force ripple to improve the electromagnetic performance of OFVA. Finally, the predicted thrust force characteristics of the LOA were validated by measurements on a prototype actuator.

\section{Design Principle and Topology of PM LOA}

Single-phase short-stroke PM LOA can be divided into two configurations [9]: C-core with one slot and E-core with two slots, as shown in Figure 2. For linear planar motion, the actuator can be cut along axial direction at any location. There are two ways to close the open coil: either only one coil winds around the stator yoke, or two coils wind around the stator teeth. The coil of C-core no longer has superiority than the coil of E-core because the winding factor will not be 1, so this paper adopts E-core quasi-Halbach magnetized topology and coil configuration.

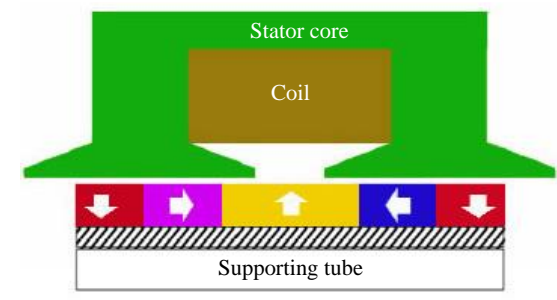

(a)

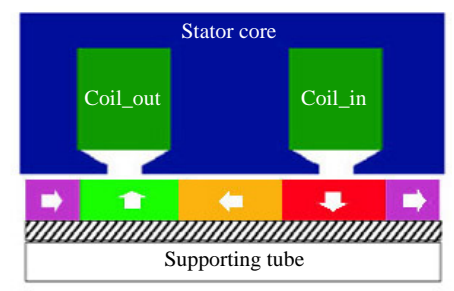

(b)

Figure 2. Topology of short stroke single-phase quasi-Halbach PM linear oscillating actuators (LOA). (a) C-core with one slot in stator; (b) E-core with two slots in stator.

\section{Influence of Stator Configuration on Thrust Force}

\subsection{Goal and Method of Comparative Design}

The preliminary design goals of the proposed actuator are to maximize its thrust force density and to minimize the ripple of thrust force. Copper loss and core loss decreased correspondingly and the efficiency improved.

The thrust force per area $f_{\rho}$, and the thrust force ripple $f_{\sigma}$, which greatly influence the operating capability of the LOA $[10,11]$, are defined as

$$
f_{\sigma}=\frac{F_{\max }-F_{\min }}{F_{\max }+F_{\min }} \times 100 \%,
$$




$$
f_{\sigma}=\frac{F_{\max }-F_{\min }}{F_{\max }+F_{\min }} \times 100 \%,
$$

Here, $F_{\text {avg }}$ is the average thrust force over the stroke, $\tau_{p}$ is the width of the unit actuator, $b_{e}$ is the added width of each side of the stator teeth, and $L_{f e}$ is the length of stator core. $F_{\max }$ is the maximum force over the stroke, and $F_{\min }$ is the minimum thrust over the stroke.

The aim of the comparative design is to find a good balance between the maximum of the thrust force density and the minimum of the thrust force ripple, and is also to control the core loss.

According to the theory of electrical machine design, the air-gap shear stress is

$$
\sigma \approx A \times B,
$$

Here, $A$ is the electrical loading of a machine, and $B$ is the magnetic loading. The permanent magnet can be designed to improve the magnetic loading [8]. Increasing the electrical loading $A$ can improve the force density of a machine. At the same time, in order to restrict the temperature rise, the force density of a machine is affected by the heat loading $q$

$$
q \approx A \times J,
$$

Here, $J$ is current density of coil. In air-cooled machines, the product $A J$ ranges from $100 \mathrm{~A}^{2} / \mathrm{mm}^{3}$ to $400 \mathrm{~A}^{2} / \mathrm{mm}^{3}$, in the case of direct water cooling, AJ can reach $2000 \mathrm{~A}^{2} / \mathrm{mm}^{3}$.

Because electrical loading $A$ appears in shear stress and heat loading at the same time, choosing a small current density and a large electrical loading can improve the thrust force density of LOA. The current density was kept at $J=4.5 \mathrm{~A} / \mathrm{mm}^{2}$ and electrical loading at $A=90 \mathrm{~A} / \mathrm{mm}$, so the product $A J$ was $405 \mathrm{~A}^{2} / \mathrm{mm}^{3}$, which is a common value in electrical machines, and the LOA can operate in air-cooled mode.

The cross-section of the designed LOA is shown in Figure 3, and the design parameters are listed in Table 1.

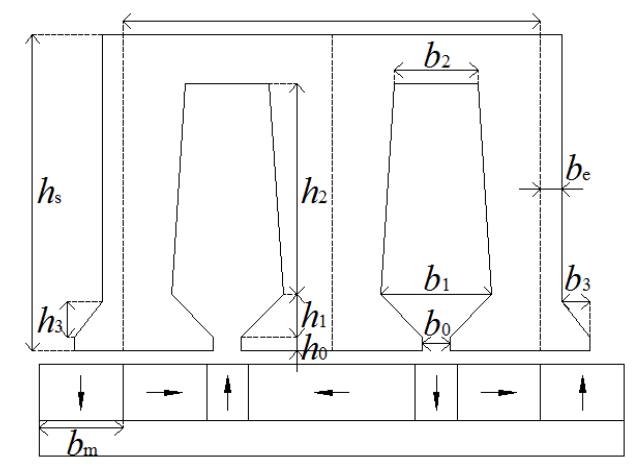

Figure 3. Cross-section of the E-cored LOA.

Table 1. Design parameters of the E-cored LOA

\begin{tabular}{cc}
\hline Design Parameter & Value \\
\hline Stator core width $2 \tau_{p}(\mathrm{~mm})$ & 64 \\
Stator core length $L_{f e}(\mathrm{~mm})$ & 50 \\
Air-gap length $g(\mathrm{~mm})$ & 2 \\
Magnet thickness $h_{m}(\mathrm{~mm})$ & 10 \\
Magnet (N38SH) remanence $B_{r}(\mathrm{~T})$ & 1.23 \\
\hline
\end{tabular}


The total current is distributed in the slot of LOA, and the needed slot area is as follows

$$
S=\frac{A \times \tau_{p}}{J \times k_{f}}
$$

Here, $k_{f}$ is the slot-fill factor and was kept to be 0.67 , so the slot area was $960 \mathrm{~mm}^{2}$. As shown in Figure 3, the slot area was defined as

$$
S=\frac{1}{2}\left[\left(b_{0}+b_{1}\right) h_{1}+\left(b_{1}+b_{2}\right) h_{2}\right],
$$

The area of the slot was kept constant, and the design parameters of the stator core were kept within a reasonable range to produce an optimal combination of parameters that could maximize the thrust force density and keep the thrust force ripple at a low level, the copper loss will keep low at the same time. Slot height $h_{s}$ is firstly considered during the optimization since $h_{s}$ has the most considerable influence on thrust force. $b_{0}$ is then considered in the process to avoid tooth tips to be saturated.

The influence of stator iron loss on the operational efficiency should not be neglected and the magnetic field distribution in the stator tooth can directly indicate iron loss, so the variations of the flux densities in stator teeth are investigated in [8]. In this way, the average flux density of tooth can be controlled under $1.5 \mathrm{~T}$ to avoid the stator teeth saturation, thereby, the stator core loss can be efficiently controlled.

\subsection{Influence of End Effects}

Actuators with E-shaped cores consist of two unit C-shaped cores and the optimal width is $2 \tau_{p}=64 \mathrm{~mm}$ [8]. End effects were not considered here during the design of the unit configuration. Therefore, a reduction would be brought to the thrust force to some extent if end effects are considered.

The configuration shown in Figure 3 was adopted to compensate for the reduction in thrust force caused by the end effects and to reduce the ripple of thrust force: two radially magnetized magnets are mounted on both sides of the mover and the E-shaped core has tooth tips on either side. The arrows represent the orientations of magnetization.

Figure 4 shows the thrust forces produced by the PM LOA over the stroke range with an ideal $\mathrm{C}$-core and with the E-core. As shown, the average thrust forces were $350 \mathrm{~N}$ and $361 \mathrm{~N}$ with and without end effects, respectively. The thrust force of ideal C-core LOA without end effects was greater than the E-core LOA with end effects, but the thrust force ripple was also greater.

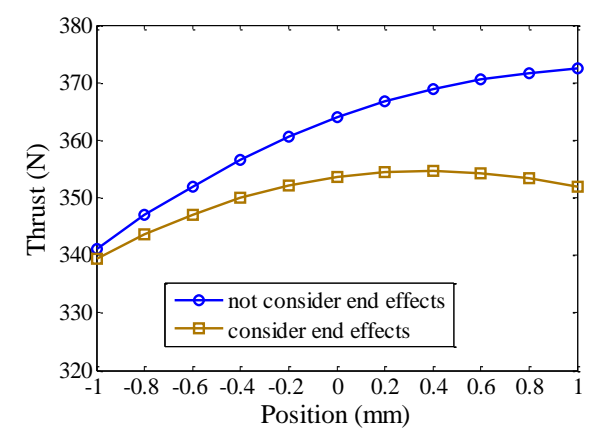

Figure 4. Thrust forces of initial LOA over the stroke range.

\subsection{Influence of Design Parameters on Thrust Force}

The design parameters of stator core—such as stator height, slot height, and slot width—were parameterized, but the slot area was kept constant. The thrust force was computed using FEA. The 
relationship between design parameter and thrust force was determined. The influence of stator teeth tip and lateral magnet on thrust force was analyzed.

Figure 5 shows the variations in thrust force and its fluctuation with stator height $h_{s}$. As shown, $h_{s}$ has considerable influence on the thrust force. The thrust force increases with $h_{s}$, and as $h_{s}$ exceeds a certain of level, it slows with increasing $h_{s}$. The fluctuation of thrust force can be minimized at an $h_{s}$ of $72 \mathrm{~mm}$. Because the magnetic circuit was not saturated, the variation of $h_{s}$ affected the flux density of stator yoke region. As $h_{s}$ increased, the flux density of stator yoke decreased. Once the flux density of the stator yoke reached a certain level and ceased to vary, the consumption of the stator core increased with $h_{S}$ and the actuator size would be great, which rendered it capable of reducing thrust force density. Finally, the stator height $h_{s}$ was determined to be $72 \mathrm{~mm}$.

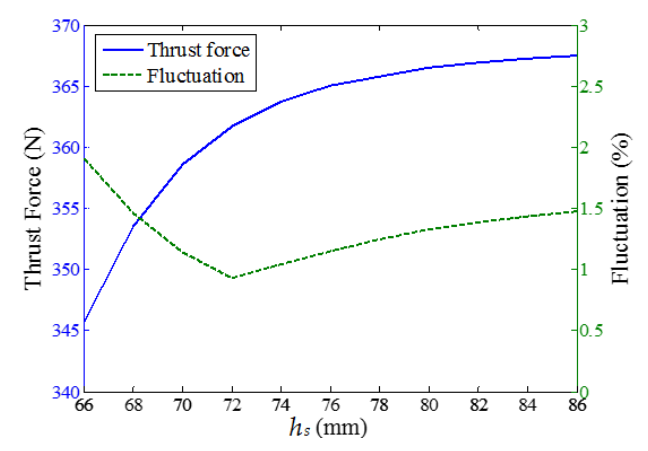

Figure 5. Variations of thrust force and its fluctuation with $h_{s}$.

Figures 6 and 7 show the variations in thrust force and their fluctuations with slot height $h_{1}$ and $h_{2}$. As shown, the thrust force first increased and then decreased, but the trends in the variation of their fluctuations followed the opposite trend. The optimal thrust force and their fluctuation was observed at an $h_{1}$ of $13 \mathrm{~mm}$ and $h_{2}$ of $48.55 \mathrm{~mm}$.

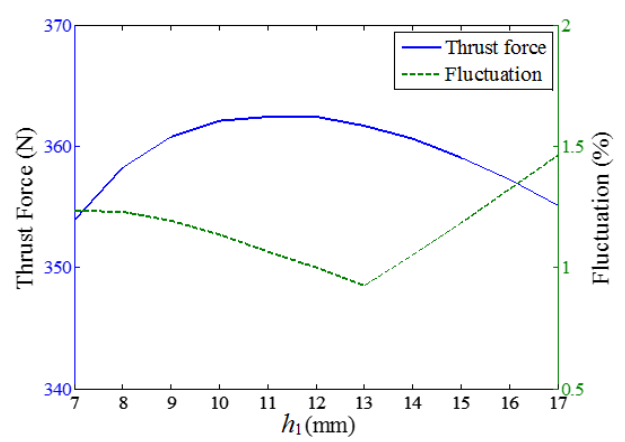

Figure 6. Variations in thrust force and its fluctuation with $h_{1}$.

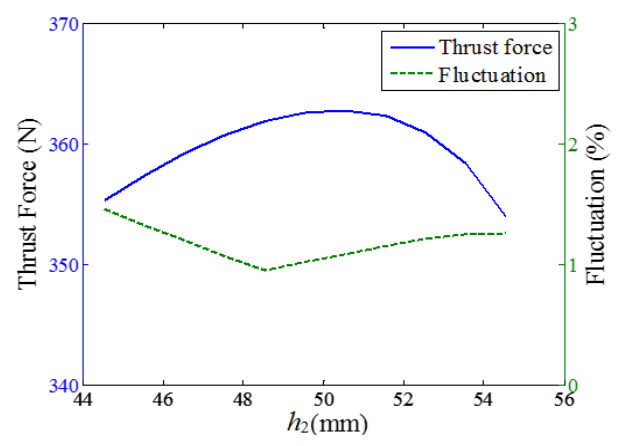

Figure 7. Variations in thrust force and its fluctuation with $h_{2}$. 
Figure 8 shows the variations in thrust force and its fluctuation with slot opening $b_{0}$. As shown, $b_{0}$ had a considerable impact. The thrust force first increased and then decreased with the $b_{0}$. Small $b_{0}$ values may cause the tooth tips to be saturated and the decrease in magnetic potential to be large, so more flux may not pass through the air gap near the stator core side; large $b_{0}$ values may cause the thrust force to fluctuate noticeably, so $b_{0}$ must be set at a reasonable value so that preferable thrust force and its fluctuation can be produced. Optimal thrust force and optimal fluctuation were observed at a $b_{0}$ of $5 \mathrm{~mm}$. The slot open width $b_{0}$ was found to be $\approx 5 \mathrm{~mm}$.

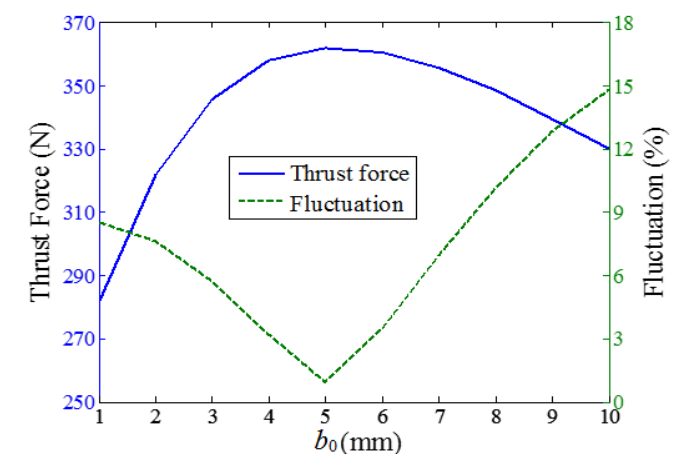

Figure 8. Variations of thrust force and its fluctuation with $b_{0}$.

Figures 9 and 10 show the variations of thrust force and its fluctuation with slot width $b_{1}$ and $b_{2}$. As shown, the thrust force first increased and then decreased, but the variation in fluctuation showed just the opposite trend. Because $b_{1}$ was $19 \mathrm{~mm}$ and $b_{2} 14.1 \mathrm{~mm}$, the optimal thrust force and its fluctuation could be determined.

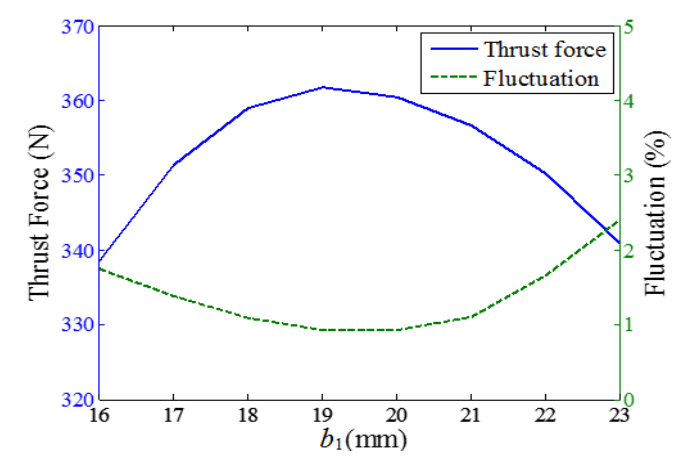

Figure 9. Variations in thrust force and its fluctuation with $b_{1}$.

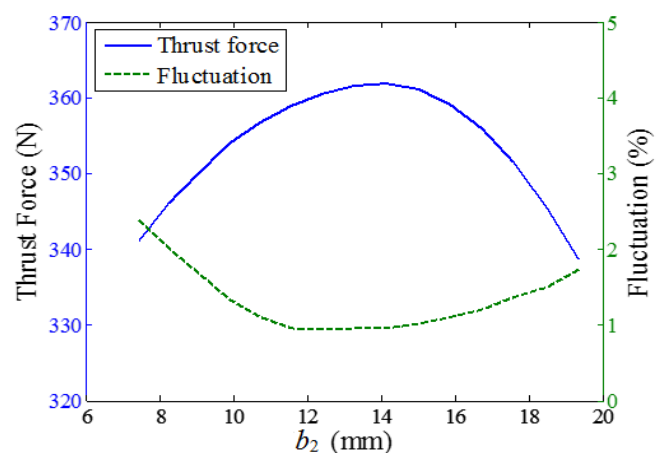

Figure 10. Variations in thrust force and its fluctuation with $b_{2}$. 
$b_{1}$ is not as identical to $b_{2}$, which was produced by the comparative design given above. For this reason, the shape of the stator tooth is unparalleled. Part of slot leakage flux passes through the slot region to form a loop and chains across some of the coils; another part traverses the slot opening to form a loop and chains across all the coils. In this way, the flux that passes through the top of the tooth region was slightly less pronounced than that of the slot root region. When the paralleled tooth was used, the tooth root region became saturated much more easily than the area surrounding the top of the tooth. The design scheme of unparalleled teeth was used because it may reduce the flux density of the tooth root region and correspondingly decrease the iron loss caused by the stator core, thus increasing actuator efficiency.

Figure 11 shows the variations of thrust force and its fluctuation with lateral tooth tip width $b_{3}$. As shown, the thrust force had a monotonically decreasing property with $b_{3}$. The thrust force fluctuation reached a minimum as $b_{3}$ approached $7 \mathrm{~mm}$. For this reason, $b_{3}$ was set to $7 \mathrm{~mm}$.

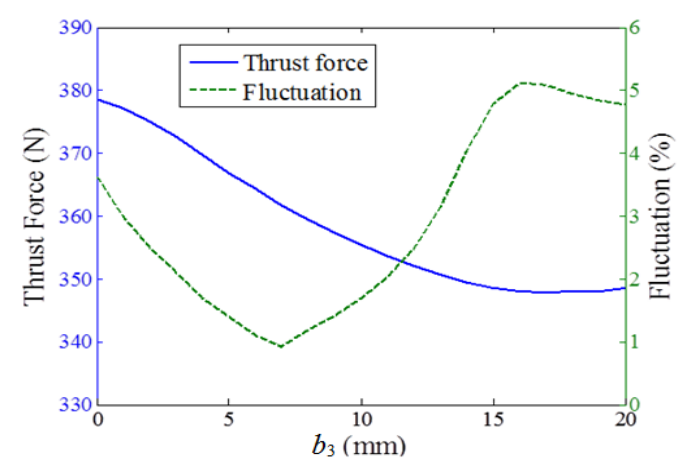

Figure 11. Variations in thrust force and its fluctuation with $b_{3}$.

Figure 12 shows the variations in thrust force and its fluctuations with stator lateral width $b_{e}$. As shown, the thrust force displayed no obvious variation as $b_{e}$ increased and its fluctuation varied by $4.8 \%$. Fluctuation was minimum at a $b_{e}$ of $0 \mathrm{~mm}$. Hence, $b_{e}$ has been chosen to be $0 \mathrm{~mm}$, which is equivalent to the structure of two $C$-cored actuators with $\tau_{p}$ being $32 \mathrm{~mm}$ combined together without additional top width of stator core.

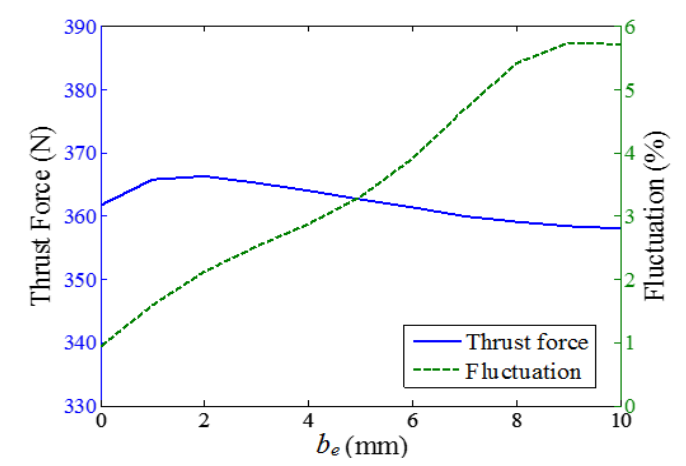

Figure 12. Variations in thrust force and its fluctuation with $b_{e}$.

Figure 13 shows the variations in thrust force and its fluctuation with $b_{m 0}$. As shown, $b_{m 0}$ had little impact on the thrust force and prominently influenced its fluctuation. The thrust force increases slowly with gradual increasing $b_{m 0}$ and its fluctuation first increased slowly and then decreased rapidly. The thrust force and its fluctuation ceased to show visible variations as $b_{m 0}$ approached $11 \mathrm{~mm}$. The lateral magnet width $b_{m 0}$ was set to $\approx 15 \mathrm{~mm}$. 


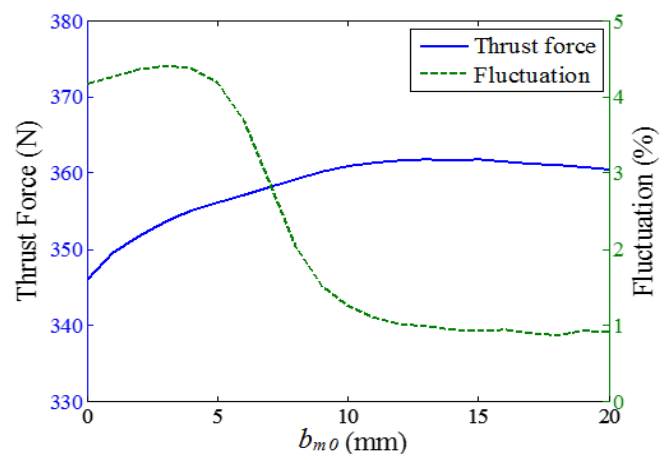

Figure 13. Variations in thrust force and its fluctuation with $b_{m 0}$.

\subsection{Influence of Design Parameters on Thrust Force}

The optimal combination of design parameters was found, and the final slot (slot A) size is given in Table 2. Another commonly used slot (slot B) with the paralleled tooth is also given for comparison.

Table 2. Design parameters of the E-core stator

\begin{tabular}{ccc}
\hline Items & \multicolumn{2}{c}{ Value } \\
\cline { 2 - 3 } & Slot A & Slot B \\
\hline Slot open width $b_{0}(\mathrm{~mm})$ & 5 & 3 \\
Slot top width $b_{1}(\mathrm{~mm})$ & 19 & 19.5 \\
Slot bottom width $b_{2}(\mathrm{~mm})$ & 14.1 & 19.5 \\
Lateral tooth tip width $b_{3}(\mathrm{~mm})$ & 7 & 7 \\
Lateral tooth width $b_{e}(\mathrm{~mm})$ & 0 & 0 \\
Lateral magnet width $b_{m 0}(\mathrm{~mm})$ & 15 & 15 \\
Tooth tip height $h_{0}(\mathrm{~mm})$ & 2 & 2 \\
Tooth top height $h_{1}(\mathrm{~mm})$ & 13 & 6 \\
Tooth bottom height $h_{2}(\mathrm{~mm})$ & 48.55 & 45.76 \\
Lateral shoulder height $h_{3}(\mathrm{~mm})$ & 9 & 9 \\
Stator height $h_{s}(\mathrm{~mm})$ & 72 & 61.9 \\
\hline
\end{tabular}

The thrust forces also changed with stroke range. As the position varied between $-1.0 \mathrm{~mm}$ and $1.0 \mathrm{~mm}$. The average thrust force of LOA with slot A and slot B was $370.3 \mathrm{~N}$ and $327.2 \mathrm{~N}$, respectively, and the thrust force ripple was $1.6 \%$ and 5\%, respectively as shown in Figure 14. The average thrust force of LOA with slot A was increased 13.2\% more than in slot B, and the thrust force ripple was $68 \%$ lower. The thrust force density of LOA with slot A increased to $115,700 \mathrm{~N} / \mathrm{m}^{2}$.

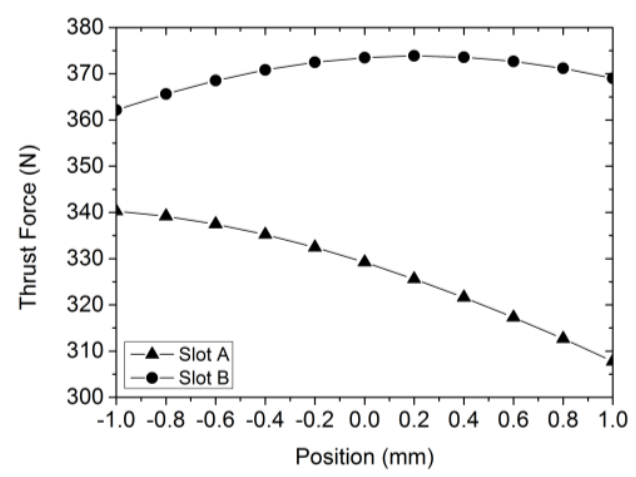

Figure 14. Variations in thrust forces with position. 


\section{Prototyping and Experiments}

A prototype has been manufactured to allow experimental validation of the analyses given above (Figure 15). The prototype is composed of various components including a stator top cap, a stator, a spring, the windings to produce an alternating magnetic field, the mover yoke, and a magnet to produce a constant magnetic field. The stator of a single-phase PM prototype is attached to a rectangular tank in a stainless steel lid. The spring that connects the stator and the mover is embedded between the lid and motor yoke. It has the function of strutting to guarantee that the air-gap length is $2 \mathrm{~mm}$. In addition, the spring should have enough stiffness to ensure that when current of stator windings is zero, the stator does leave its initial position $(x=0, y=0)$. When the mover is fixed, the stator can repeat a reciprocating motion under the action of the electromagnetic force. The schematic diagram of experimental device is shown in Figure 16.

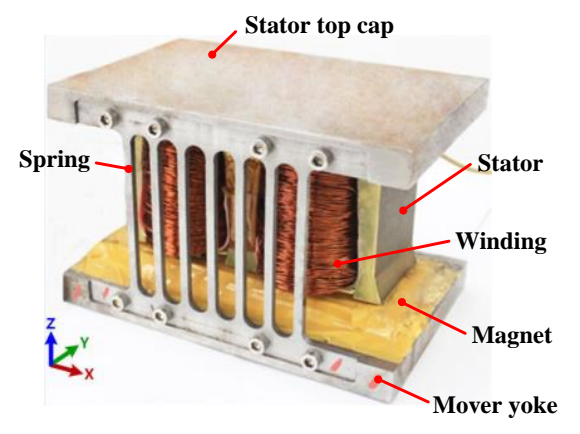

Figure 15. Prototype LOA.

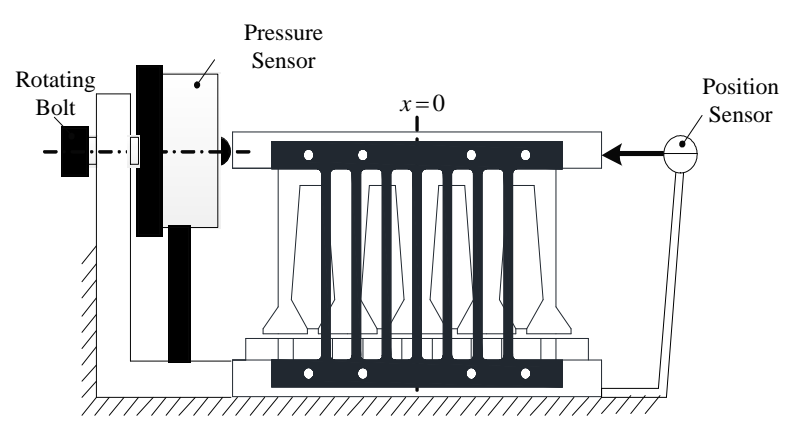

Figure 16. Schematic diagram of experimental device.

The initial position of the stator is measured using a position sensor under the condition that the current of stator windings is zero. When a DC voltage is applied to the windings, the stator is forced to move leftward by the electromagnetic force accordingly. Meanwhile, the stator windings are also subjected to a rightward spring force. The spring force will decline if the stator is subjected to another rightward force generated by a rotating bolt and it will decrease to zero when the stator returns to its initial position. Then the output voltage can be measured by the pressure sensor while the current can be recorded according to the DC power supply. Thus, the electromagnetic force to which stator windings are subjected at the initial position can be calculated by converting the output voltage into the corresponding force. By changing the applied DC voltage and repeating the aforementioned steps, a figure can be drawn that depicts the changes in electromagnetic force with current.

The predicted thrust force-current characteristics (Figure 17) are compared and measured. As shown, there was strong agreement between the FE predicted, and results were measured. When the figure of ampere-turn was between 0 and $2500 \mathrm{~A}$, there was a linear relationship between the thrust force and the ampere-turn. However, when the figure of ampere-turn was over $2500 \mathrm{~A}$, the two factors showed a nonlinear relationship because of the magnetic saturation in the actuator. The increase in 
the temperature of the prototype winding was only $43{ }^{\circ} \mathrm{C}$, which indicated that the quasi-Halbach magnetized LOA had superior overload performance.

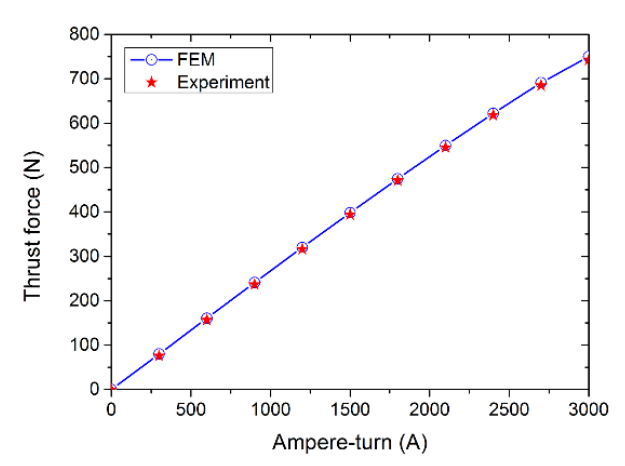

Figure 17. Variations in thrust forces with ampere-turns.

\section{Conclusions}

To achieve the desirable performance of OFVA, an E-cored quasi-Halbach magnetized LOA has been comparatively designed and its electromagnetic performances were analyzed by FEA. The optimal design parameters have been presented, the thrust force density was increased, and the thrust force ripple was reduced. Predicted and measured results showed strong agreement.

Comparative design of the stator can improve thrust force density and reduce thrust force ripple of LOA. Reducing the current density while increasing electrical loading can increase the thrust force density. Using a stator with unparalleled teeth and increased slot height can increase the thrust force density. The slot's open width was found to have a significant effect on the thrust force density and thrust force ripple.

Acknowledgments: This work is supported by National Key Basic Research Program of China under Grant 2013CB035600.

Author Contributions: J.H. and M.Z. conceived and designed the experiments; J.H. and Y.L. performed the experiments; J.H. and J.Z. wrote the paper.

Conflicts of Interest: The authors declare no conflict of interest.

\section{References}

1. Chen, L.; Li, W.Y.; Ma, T.J. The state of the art and perspectives of linear friction welding technology. Adv. Aeronaut. Sci. Eng. 2010, 1, 178-183.

2. Lee, H.K.; Song, G.Y.; Park, J.S.; Hong, E.P.; Jung, W.H.; Park, K.B. Development of a linear motor for compressors of household refrigerators. In Proceedings of the International Compressor Engineering Conference, West Lafayette, IN, USA, 25-28 July 2000; pp. 283-286.

3. Goto, A.; Okamoto, T.; Ikariga, A.; Todaka, T.; Enokizono, M. A New Moving-magnet Type Linear Actuator utilizing Flux Concentration Permanent Magnet Arrangement. J. Electr. Eng. Technol. 2012, 3, 342-348. [CrossRef]

4. Hong, S.K.; Ro, J.S.; Jung, H.K. Optimal Design of a Novel Permanent Magnetic Actuator using Evolutionary Strategy Algorithm and Kriging Meta-model. J. Electr. Eng. Technol. 2014, 9, 471-477. [CrossRef]

5. Lu, Q.F.; Yu, M.H.; Ye, Y.Y.; Fang, Y.T.; Zhu, J.G. Thrust Force of Novel PM Transverse Flux Linear Oscillating Actuators With Moving Magnet. IEEE Trans. Magn. 2011, 47, 4211-4214. [CrossRef]

6. Fujimoto, Y.; Kominami, T.; Hamada, H. Development and Analysis of a High Thrust Force Direct-Drive Linear Actuator. IEEE Trans. Ind. Electr. 2009, 56, 1383-1392. [CrossRef]

7. Wang, J.B.; Atallah, K.; Wang, W.Y. Analysis of a Magnetic Screw for High Force Density Linear Electromagnetic Actuators. IEEE Trans. Magn. 2011, 47, 4477-4480. [CrossRef] 
8. Xu, F.; Hu, J.H.; Zou, J.B.; Li, Y.; Xu, Y.X.; Fan, H. Comparative investigation of permanent magnet linear oscillatory actuators used in orbital friction vibration machine. Int. Symp. Appl. Electromagn. Mech. 2014, 45, 581-588.

9. Wang, J.; Howe, D. A tubular permanent magnet machine equipped with homopolar windings. In Proceedings of the International Symposium on Linear Drives for Industry Applications (LDIA 2005), Kobe-Awaji, Japan, 25-28 September 2005; pp. 239-242.

10. Yeo, H.K.; Woo, D.K.; Lim, D.K.; Ro, J.S. Analysis of a Surface-Mounted Permanent-Magnet Machine with Overhang Structure by Using a Novel Equivalent Magnetic Circuit Model. J. Electr. Eng. Technol. 2014, 9 , 1960-1966. [CrossRef]

11. Ahn, H.M.; Oh, Y.H.; Song, K.D.; Kim, Y.I.; Kho, H.R.; Choi, M.S.; Hahn, S.C. Optimal Design of Permanent Magnetic Actuator for Permanent Magnet Reduction and Dynamic Characteristic Improvement using Response Surface Methodology. J. Electr. Eng. Technol. 2015, 10, 935-943. [CrossRef]

2017 by the authors. Licensee MDPI, Basel, Switzerland. This article is an open access article distributed under the terms and conditions of the Creative Commons Attribution (CC BY) license (http://creativecommons.org/licenses/by/4.0/). 